Proceedings

\title{
Remotely-Delivered Mind-Body Physical Activity Improves Physical Function in Older Adults
}

\author{
Lesley-Anne Tanhamira, Gurch Randhawa and David Hewson *
}

Institute for Health Research, University of Bedfordshire, Luton, UK; lesley-anne.tanhamira@study.beds.ac.uk, gurch.randhawa@beds.ac.uk

* Correspondence: david.hewson@beds.ac.uk

\begin{abstract}
A pilot trial of remotely delivered mind-body physical activity was carried out in the UK during the COVID-19 pandemic. Participants performed twice weekly one-hour sessions of the program for eight weeks, with sessions delivered using Zoom. Physical function was evaluated using the Short Physical Performance Battery (SPPB), with the individual components of balance, gait speed and the Five-times Sit-To-Stand (5STS) also compared. A significant improvement was noted in 5STS performance for the experimental group $(n=10)$ of 4.1 seconds $(d=2.0 ; 95 \%$ CI: $0.8,3.2)$. There were no significant differences for balance, gait speed or total SPPB score. Future work is needed to determine whether remotely delivered physical activities could be a feasible alternative to face-toface sessions for older people with limited mobility.
\end{abstract}

Keywords: remote physical activity; mind-body exercise; older people; physical function; sit-tostand

\section{Introduction}

As people get older, there is an increase in loneliness, isolation and poorer health outcomes, with these problems attributed to a decline in physical, cognitive, or social reserves [1]. Active living encourages adults to be more physically active, which in turn can reduce the severity of disability and falls risk in older people, as well as improve mental and social wellbeing [2]. Activities for older people were found to have better engagement if they were enjoyable and had less focus on participation for longer term health outcomes, but instead focused on short term benefits and included a sociable element for participants [3]. Mind-body exercises such as yoga and tai chi have been shown to improve cognition, physical function and quality of life in older people [4]. However, people with limited mobility, or chronic pain may not be able to participate due to physical limitations [5]. Adapted versions of these exercises, such as seated exercises instead of standing are becoming more common; and although there is a wide evidence base for mind body exercise

Publisher's Note: MDPI stays neutral with regard to jurisdictional claims in published maps and institutional affiliations.

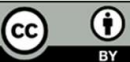

Copyright: $(2020$ by the authors. Submitted for possible open access publication under the terms and conditions of the Creative Commons Attribution (CC BY) license (http://creativecommons.org/licenses/by/4.0/). $[6,7]$, the effectiveness of adapted mind body physical activity program remains limited.

Barriers to maintaining physical activity in the older population include social inequalities, culture, and even physical abilities [8]. Older people with limited mobility, or those who struggle to access community events are at a disadvantage, as access to exercise classes could become a challenge due to issues such as poor transport links or poor financial resources to cover transport costs [9]. These problems have been exacerbated during the COVID-19 pandemic, particularly for older people who have been reported to be at higher risk and have therefore been advised to shield and self -isolate (Amon, 2020). In such cases, face-to-face exercise classes were no longer possible, which has made remotely delivered services an important means for people to maintain access to different resources whilst adhering to the social distancing and social isolation guidelines [10]. The use of video conferencing platforms has included not only video calls, but also church services, birthday parties and exercise classes [11]. The use of different technologies has been 
reported to positively impact on the wellbeing of people as it improves social inclusion, with a growing evidence base for the effectiveness of technologies such as telecare and telehealth in improving health outcomes [12].

The aim of this pilot study is to evaluate whether a mind-body exercise program delivered remotely using a video-conferencing platform can positively impact on the physical function of older participants. In addition, this pilot study will evaluate whether remotely delivered mind-body exercise is acceptable and feasible and could potentially be used after the COVID-19 crisis to increase access to physical activity programs for older people with difficulty attending face-to-face classes.

\section{Materials and Methods}

\subsection{Participants}

Convenience sampling was used to recruit participants using social media adverts and emails sent to people on the mailing list of the Letchworth Centre of Healthy Living (www.letchworthcentre.org), which is a charity based in Hertfordshire, UK. The inclusion criteria included being aged 65 years and over, community-dwelling and having internet access and video conferencing capabilities. Participants were excluded if they had dementia, were unable to provide consent or had contraindications to adaptive yoga. Participants were screened during an initial telephone consultation, and if eligible, a zoom video call was arranged. Informed consent was provided by the participants before the initial assessments. Ethical approval for the study was granted by the Institute for Health Research Ethics Committee at University of Bedfordshire (IHREC939).

\subsection{Study Design}

The study was a quasi-experimental trial, with participants randomly allocated to a wait-listed control group or to the experimental group. Recruitment occurred in May 2020 while the UK was in a lockdown due to the effects of the first wave of COVID-19, with the trial running from June to October 2020. Data collection occurred remotely over Zoom video conferencing software (Zoom Video Communications Inc, San Jose, CA, USA). The physical activity sessions were also conducted over zoom by yoga instructor with over 20 years of experience. All outcome measures were assessed at baseline and at eight weeks at the end of the intervention. After consultation with the instructor, each group was limited to 10 to ensure the instructor could interact with all participants.

\subsection{Mind-Body Physical Activity Intervention}

The intervention, known as the Positive Movement Program (PMP), was developed by the Letchworth Centre of Healthy Living and is based on adaptive yoga, which has been adapted appropriately for older adults (Tew et al., 2017). These sessions include exercises that focus on key elements of yoga, including asana (posture), pranayama (breath work), and dharana (mental focus). Positive Movement also includes exercises from Tai Chi and Chi Kung, as well as the Alexander Technique for posture exercises and elements of mindfulness. Each session lasts up to 60 minutes, with two sessions performed each week. Each class began with an introduction to the class and finished with 15 minutes of socializing amongst the participants. Examples of some of the exercises are shown in Figure 1 below.

\subsection{Outcome Measures}

The primary outcome measure was the Short Physical Performance Battery (SPPB), which is a composite test that contains measures of balance, gait speed measured over 8feet $(2.57 \mathrm{~m})$ and the Five-times Sit-To-Stand (5STS) chair test [13]. Each of the individual tests is given a score from 0 to 4 , with all three scores added together to give an overall score from 0-12. Scores from 10-12 indicate high function, with 7-9 classified as moderate function, while scores of $<6$ indicate low function. For the individual tests of gait speed 
and the 5STS, the values of these tests were used rather than the scores for the SPPB to eliminate any ceiling effects caused by the thresholds for SPPB scores. All measurements were taken remotely using Zoom, with timed performance for gait speed and 5STS measured using the time of the video recording from Zoom and verified with a handheld stopwatch.

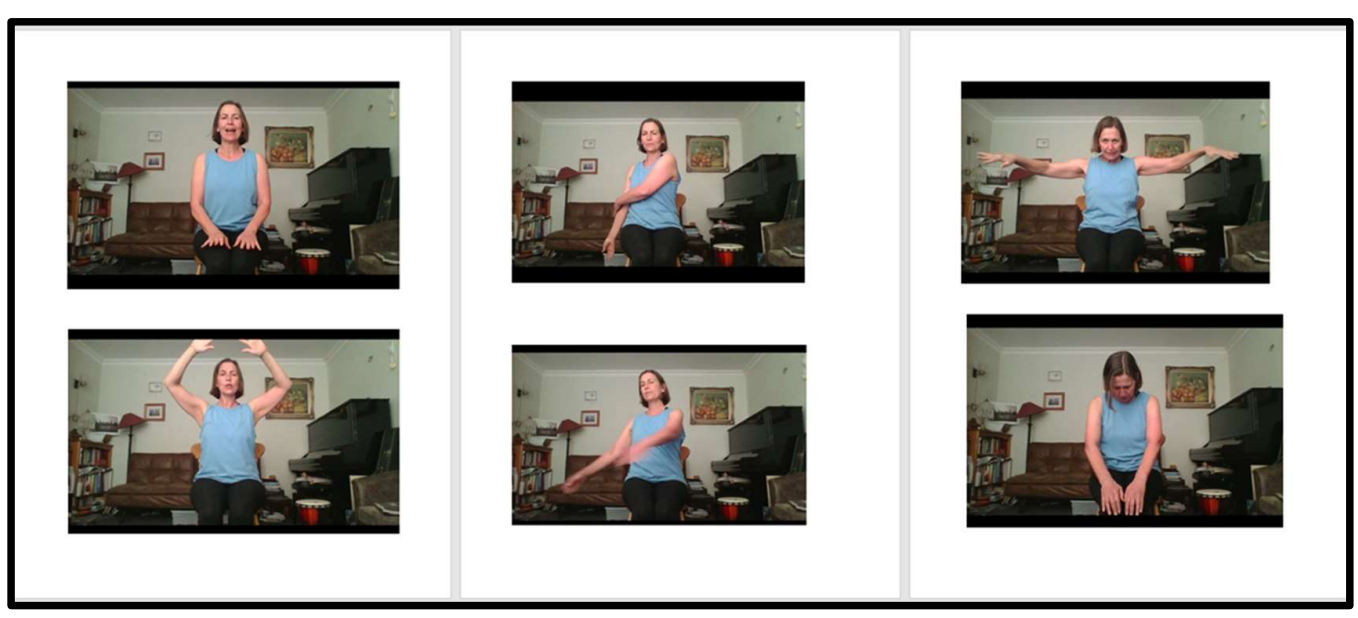

Figure 1. Sample of exercises from a session of the Positive Movement Program.

In addition to physical function, participants were screened for frailty before the trial, using the FRAIL scale, which is a simple 5-question scale that can be administered without requiring any face to face measurements to be obtained [14].

\subsection{Statistical Analysis}

The data was analyzed using IBM SPSS Statistics Version 26 (IBM Corporation, Armonk, NY, USA). A paired t-test was used to compare pre- and post-scores for each group, with 95\% confidence limits around the mean reported [15]. ANOVA was used to compare differences in the pre-post changes between groups.

\section{Results}

\subsection{Participants}

The characteristics of the ten participants in each group are shown in Table 1. During the trial, one participant in the intervention group and two participants in the control group withdrew from the trial due to reasons unrelated to the trial. In the experimental group, a participant dropped out due to ongoing health problems, whilst in the control group the two dropouts were due to personal reasons and due to back problems, which made it difficult for them to do chair exercises for prolonged periods. In total, 17 participants completed the study (85\%), with all intervention participants attending at least half the sessions over the eight-week intervention period.

Table 1. Participant characteristics.

\begin{tabular}{ccc}
\hline & Intervention & Control \\
\hline Age (years) $^{1}$ & $68.1 \pm 6.5$ & $69.2 \pm 5.9$ \\
Sex $^{2}$ & $8(80)$ & $9(90)$ \\
${\text { Height }(\mathrm{m})^{1}}^{1}$ & $1.67 \pm 0.11$ & $1.66 \pm 0.07$ \\
${\text { Weight }(\mathrm{kg})^{1}}^{1}$ & $66.6 \pm 13.5$ & $74.6 \pm 10.8$ \\
FRAIL score $^{1}$ & $0.6 \pm 0.7$ & $1.0 \pm 1.1$ \\
Fit $^{2}$ & $7(70)$ & $6(60)$ \\
Pre-frail $^{2}$ & $3(30)$ & $3(30)$ \\
\hline
\end{tabular}




\begin{tabular}{|c|c|c|}
\hline Frail 2 & $0(0)$ & $1(10)$ \\
\hline
\end{tabular}

Pre and post scores for the physical function outcomes for the two groups are shown in Table 2. There was a significant improvement in both 5STS time and 5STS score for the intervention group after the eight-week trial, but no other significant differences were observed. When the pre-post changes were compared between groups, there was a significant improvement of 4.1 seconds for 5STS for the experimental condition, when compared to the controls. This equates to a large effect size (Cohen's $d=2.0 ; 95 \% \mathrm{CI}: 0.8,3.2)$. No significant differences were observed for balance $(\mathrm{d}=0.2 ;-0.5,1.5)$, gait speed $(\mathrm{d}=-0.1$; $1.3,1.1)$, or for the total SPPB score $(\mathrm{d}=0.9 ;-0.1,1.9)$.

Table 2. Physical function of intervention and control groups before and after the eight-week trial.

\begin{tabular}{ccccc}
\hline \multirow{2}{*}{ Outcome } & \multicolumn{2}{c}{ Intervention } & \multicolumn{2}{c}{ Control } \\
& Pre & Post & Pre & Post \\
\hline Balance score $^{1}$ & $3.6(3.3,4.0)$ & $3.6(3.3,4.0)$ & $3.2(2.5,3.8)$ & $3.0(2.2,3.7)$ \\
Gait speed (m/s) $^{1}$ & $0.52(0.44,0.62)$ & $0.50(0.39,0.61)$ & $0.41(0.35,0.47)$ & $0.41(0.34,0.47)$ \\
Gait score $^{1}$ & $2.0(1.5,2.5)$ & $1.8(1.3,2.5)$ & $1.3(1.1,1.6)$ & $1.4(1.2,1.7)$ \\
5STS time (sec) $^{1}$ & $19.2(15.3,23.3)$ & $16.7(13.2,20.5)^{*}$ & $17.5(11.8,25.7)$ & $19.2(12.6,29.1)$ \\
5STS score $^{1}$ & $1.6(1.0,2.3)$ & $2.3(1.4,3.1)^{*}$ & $2.2(1.5,3.0)$ & $2.2(1.6,3.0)$ \\
SPPB score $^{1}$ & $7.3(6.0,8.7)$ & $7.8(6.3,9.3)$ & $6.8(5.8,7.7)$ & $6.7(5.3,7.9)$ \\
SPPB 10-12 $^{2}$ & $2(25)$ & $2(25)$ & $0(0)$ & $0(0)$ \\
SPPB 7-9 ${ }^{2}$ & $2(25)$ & $3(37.5)$ & $5(55.6)$ & $5(55.6)$ \\
SPPB 0-6 ${ }^{2}$ & $4(50)$ & $3(37.5)$ & $4(44.4)$ & $4(44.4)$ \\
\hline
\end{tabular}

${ }^{1}$ Data are means and 95\% confidence intervals; ${ }^{2}$ Data are $\mathrm{n}(\%) ;{ }^{*}$ Significant difference pre vs. post.

\section{Discussion}

The adherence rates for the intervention group indicated that remote delivery of physical activities can be conducted successfully using video conferencing software. Participants were able to log in and use the Zoom platform with minimal disruption to the sessions, indicating that remote delivery could be a feasible option for increasing access to physical activity programs in the older population regardless of prior technology competency levels. It should be noted that all participants in the intervention group were classified before the trial as fit or pre-frail using the FRAIL scale [14], therefore, it might have been more manageable for them to access zoom sessions than frail older people. However, when the SPPB categories were examined, most of the intervention group could be considered as having low-moderate physical function, with increased risk of falls [16]. Further studies are required on a larger population group including those with less experience of technology and a greater range of frailty levels.

The intervention group showed a significant improvement in lower extremity strength after eight weeks, as measured using the 5STS. Such an improvement in strength would be likely to decrease the risk of falls in this group and potentially improving participants balance and balance confidence, although no changes in balance scores were observed. One reason for this improvement could be the type of exercises conducted during the trial, with most movements chair-based and graduating to standing, focusing a lot on lower strength and balance. Although no significant results were shown for the balance component of the SPPB, this could have been due to the ceiling effects of this component. The highest score on the balance test was a ' 4 ' indicating the participant can complete all sections of the balance test for up to 10 seconds. All of the intervention group scored within the ' 3 ' to ' 4 ' range at baseline, showing they were already reaching the highest or close to the highest possible score on this outcome measure. Further analysis using more 
precise measures of balance are warranted to deduce if any improvements in balance would occur following exposure to the intervention. There were no improvements in gait speed, however this observation is not surprising as the intervention mostly focuses on strength and balance. Finally, there were no significant changes in total SPPB score, as only the 5STS component improved. In addition, on closer observation, although there was an overall improvement in 5STS time, many of the participants had initial times that were much slower than the lowest threshold level, meaning that although they improved, they were still classified in the same level after the eight-week trial. It is likely that a longer intervention might produce greater changes in SPPB scores.

The role of physical activity in the older population is widely documented as being crucial to maintaining positive health outcomes [17]. Furthermore, mind body exercises aim to address social and psychological factors that improve health outcomes [4,6,7], with these physical even more essential during the COVID-19 pandemic. Although this was a pilot study to measure the effectiveness of the remotely delivered program on physical function in older people, the study also allowed the authors to assess if remote delivery was a feasible way to deliver physical activity to older people. The study showed that the SPPB assessment can be conducted online, however, a limitation of the study is that it was not possible to validate the SPPB measurement as face-to-face assessment was not possible. It is possible that delays in video transmission or problems due to bad internet connections could change the results. A validity study is planned to determine the accuracy of the timed tests, although this study can only be carried out once the COVID-19 guidelines have changed to make it safe to do so. This validity study that will compare measures collected remotely with data collected face to face on the same participants.

The remote delivery of PMP allowed participants to have access to physical activity sessions throughout the 2020 COVID-19 pandemic. Future work is planned in 2021 as the COVID-19 pandemic continues, with remote PMP evaluated out on a larger scale to allow a more varied group of the population to have access to physical activities that can positively impact their physical function. In addition to serving older adults during the pandemic, this mode of delivery can allow participants with limited mobility, those living in restricted geographical locations, or those who simply prefer to stay within their own homes, a chance to access and participate in group exercises provided they have internet access and capable devices. Future studies within this area are needed to reinforce these findings.

Author Contributions: Conceptualization, L.T., G.R and D.H.; methodology, L.T and D.H.; formal analysis, L.T and D.H.; investigation, L.T.; data curation, L.T.; writing - original draft preparation, L.T.; writing - review and editing, D.H. All authors have read and agreed to the published version of the manuscript.

Funding: This research was conducted as part fulfillment of a PhD program of LT, which is funded by The Letchworth Centre for Healthy Living.

Acknowledgments: The authors would like to thank the instructor and the participants for their support.

Conflicts of Interest: The authors declare no conflict of interest. The funders had no role in the design of the study; in the collection, analyses, or interpretation of data; in the writing of the manuscript, or in the decision to publish the results.

\section{References}

1. Kyu, H.H.; Abate, D.; Abate, K.H.; Abay, S.M.; Abbafati, C.; Abbasi, N.; Abbastabar, H.; Abd-Allah, F.; Abdela, J.; Abdelalim, A. Global, regional, and national disability-adjusted life-years (DALYs) for 359 diseases and injuries and healthy life expectancy (HALE) for 195 countries and territories, 1990-2017: a systematic analysis for the Global Burden of Disease Study 2017. Lancet 2018, 392, 1859-1922.

2. World Health Organization. Ageing and Health Key Facts; 2018.

3. Devereux-Fitzgerald, A.; Powell, R.; Dewhurst, A.; French, D.P. The acceptability of physical activity interventions to older adults: A systematic review and meta-synthesis. Soc Sci Med 2016, 158, 14-23. 
4. Brosseau, L.; Taki, J.; Desjardins, B.; Thevenot, O.; Fransen, M.; Wells, G.A.; Imoto, A.M.; Toupin-April, K.; Westby, M.; Gallardo, I.C.Á. The Ottawa panel clinical practice guidelines for the management of knee osteoarthritis. Part one: introduction, and mind-body exercise programs. Clin Rehabil 2017, 31, 582-595.

5. Khuzema, A.; Brammatha, A.; Arul Selvan, V. Effect of home-based Tai Chi, Yoga or conventional balance exercise on functional balance and mobility among persons with idiopathic Parkinson's disease: An experimental study. Hong Kong Physiother J 2020, 40, 39-49.

6. Wu, C.; Yi, Q.; Zheng, X.; Cui, S.; Chen, B.; Lu, L.; Tang, C. Effects of mind-body exercises on cognitive function in older adults: A meta-analysis. J Am Geriatr Soc 2019, 67, 749-758.

7. Zou, L.; Loprinzi, P.D.; Yu, J.J.; Yang, L.; Li, C.; Yeung, A.S.; Kong, Z.; Chiou, S.-Y.; Xiao, T. Superior Effects of Modified ChenStyle Tai Chi versus 24-Style Tai Chi on Cognitive Function, Fitness, and Balance Performance in Adults over 55. Brain Sciences 2019, 9, doi:10.3390/brainsci9050102.

8. Cheval, B.; Rebar, A.L.; Miller, M.W.; Sieber, S.; Orsholits, D.; Baranyi, G.; Courvoisier, D.; Cullati, S.; Sander, D.; Chalabaev, A. Cognitive resources moderate the adverse impact of poor perceived neighborhood conditions on self-reported physical activity of older adults. Prev Med 2019, 126, 105741.

9. Franco, M.R.; Tong, A.; Howard, K.; Sherrington, C.; Ferreira, P.H.; Pinto, R.Z.; Ferreira, M.L. Older people's perspectives on participation in physical activity: a systematic review and thematic synthesis of qualitative literature. Br J Sports Med 2015, 49, $1268-1276$.

10. Ng, K. Adapted physical activity through COVID-19. Eur J Adapt Phys Act 2020, 13, 1-1, doi:10.5507/euj.2020.003.

11. Berg-Weger, M.; Morley, J. Editorial: Loneliness and social isolation in older adults during the Covid-19 pandemic: Implications for gerontological social work. J Nutr Health Aging 2020, 24, 456-458, doi:doi.org/10.1007/s12603-020-1366-8.

12. Chopik, W.J. The benefits of social technology use among older adults are mediated by reduced loneliness. Cyberpsychol Behav Soc Netw 2016, 19, 551-556.

13. Guralnik, J.M.; Simonsick, E.M.; Ferrucci, L.; Glynn, R.J.; Berkman, L.F.; Blazer, D.G.; Scherr, P.A.; Wallace, R.B. A short physical performance battery assessing lower extremity function: association with self-reported disability and prediction of mortality and nursing home admission. J Gerontol 1994, 49, M85-M94, doi:10.1093/geronj/49.2.M85.

14. Morley, J.E.; Malmstrom, T.K.; Miller, D.K. A simple frailty questionnaire (FRAIL) predicts outcomes in middle aged African Americans. J Nutr Health Aging 2012, 16, 601-608, doi:10.1007/s12603-012-0084-2.

15. Gardner, M.J.; Altman, D.G. Confidence-intervals rather than P values: estimation rather hypothesis testing. Br. Med. J. 1986, 292, 746-750, doi:10.1136/bmj.292.6522.746.

16. Veronese, N.; Bolzetta, F.; Toffanello, E.D.; Zambon, S.; De Rui, M.; Perissinotto, E.; Coin, A.; Corti, M.C.; Baggio, G.; Crepaldi, G., et al. Association between Short Physical Performance Battery and falls in older people: the Progetto Veneto Anziani Study. Rejuvenation Res 2014, 17, 276-284, doi:10.1089/rej.2013.1491.

17. Cruz-Jentoft, A.J.; Woo, J. Nutritional interventions to prevent and treat frailty. Curr Opin Clin Nutr Metab Care 2019, 22, 191195. 